\title{
A Method of Hydrophobically Modifying Paper with a Trace Reagent
}

\author{
Jingjing Zhou, ${ }^{\mathrm{a}}$ Guowei Gao, ${ }^{\mathrm{a}}$ Yansheng Li, ${ }^{\mathrm{a}, *}$ Dongdong Wang, ${ }^{\mathrm{b}, *}$ Jingfang Hu, ${ }^{\mathrm{a}, \mathrm{d}}$ \\ Yu Song, ${ }^{\mathrm{a}}$ and Peng Chen ${ }^{\mathrm{c}, *}$
}

\begin{abstract}
It is of great importance to protect paper cultural relics, especially in terms of making them waterproof. One of the important ways to protect paper cultural relics is by hydrophobic modification treatment. However, most of the existing hydrophobic modification methods rely on a great deal of organic solvents, which poses greater risks to safety and pollution. In this work, a simple, green, and effective method for paper hydrophobic treatment was proposed and evaluated. The vapor of a fluorinated triethoxysilane was reacted with the active hydroxyl group on the paper in a closed space, which realized the hydrophobic modification of the paper in one step. The contact angle of the hydrophobic paper prepared by this method was $148.4^{\circ}$ under optimal experimental conditions. In addition, this method did not damage the paper, based on the results of the scanning electron microscopy of the paper before and after hydrophobic treatment. The generality of this method was verified by using other substrates and hydrophobic reagents. This method is simple to operate and does not require the usage of a large amount of organic reagent. It is expected to receive widespread attention in the field of the protection of paper and wooden cultural relics.
\end{abstract}

DOI: 10.15376/biores.17.1.384-399

Keywords: Hydrophobic; Surface modification; Filter paper; Trace reagent

Contact information: a: Beijing Key Laboratory for Sensors, Beijing Information Science \& Technology University, Beijing 100101 P.R. China; b: Institute of cosmetic regulatory science, Beijing Technology \& Business University Beijing 100048 P.R. China; c: Beijing Key Laboratory of Traditional Chinese Medicine Basic Research on Prevention and Treatment for Major Diseases, Experimental Research Center, China Academy of Chinese Medical Sciences, Beijing 100700 P.R. China; d: State Key Laboratories of Transducer Technology, Shanghai Institute of Microsystems and Information Technology, Chinese Academy of Sciences, Shanghai 200050 P.R. China;

* Corresponding author: lys2019@bistu.edu.cn

\section{INTRODUCTION}

Paper cultural relics are an important part of cultural heritage and have substantial cultural, scientific, and artistic value (Chen et al. 2016; Liu et al. 2019). However, paper cultural relics have been undergoing severe deterioration and aging problems over time (Xu et al. 2020; Zhang et al. 2020). Temperature and humidity are the most important indicators of the environmental protection of paper cultural relics, among the many environmental factors that affect paper cultural relics. As such, excessive humidity will cause paper cultural relics to suffer from handwriting fading and paper adhesion, as well as generate harmful organisms (Zervos and Alexopoulou 2015; Xu 2018). The methods of controlling the temperature and humidity rely on central air conditioning and constant temperature and humidity equipment (Wen 2020). However, the conservation of cultural 
relics is still affected by a hot and humid climate, due to insufficient operating time of the control equipment, an unsatisfactory control effect, equipment failure, and other reasons. For this reason, a relatively stable and suitable humidity cannot be guaranteed during transportation (Lazaridis et al. 2018). The damage of paper cultural relics caused by these factors will cause irreversible harm to historical and cultural values. The strategy of relying on equipment to regulate temperature and humidity is still insufficient for the protection of paper cultural relics. Therefore, the hydrophobic treatment method is one of the effective methods to protect paper cultural relics against the effects of wetting by liquid water, which can help avoid the damaging of paper cultural relics due to humidity changes (Reyden 1992).

There are many methods and technologies for the hydrophobic surface modification of paper, including the spraying method and the dipping method (Li et al. 2006, 2007; Xue et al. 2014; Li et al. 2015, 2016; Wang et al. 2016; Li et al. 2021). Zhang et al. (2012) sprayed a prepared modified solution onto the surface of filter paper to form a superhydrophobic filter paper. Firstly, the $\mathrm{SiO}_{2}$ particles were prepared via the sol-gel method and then modified with octadecyltrichlorosilane. Then, polystyrene was completely dissolved in tetrahydrofuran. Finally, the modified $\mathrm{SiO}_{2}$ particles were dispersed in the tetrahydrofuran to prepare the modified solution. In the process of synthesizing the modified $\mathrm{SiO}_{2}$ particles, it took $12 \mathrm{~h}$ of magnetic stirring and $12 \mathrm{~h}$ of vacuum drying after adding the octadecyltrichlorosilane. Musikavanh et al. (2019) proposed a three-step method to prepare superhydrophobic cellulose filter paper. Firstly, the filter paper was pre-treated with zinc chloride to activate the cellulose fibers. Secondly, $\mathrm{SiO}_{2}$ was coated on the surface of filter paper to increase the roughness, and then the filter paper was dried for $12 \mathrm{~h}$. Finally, the filter paper was soaked in a $3.5 \%(\mathrm{w} / \mathrm{v})$ hexadecyltrimethoxysilane and ethanol solution for $2 \mathrm{~h}$. The contact angle of the modified filter paper was $154.8^{\circ}$. Liu et al. (2018) developed a method of modifying filter paper based on the polymethylhydrogensiloxane dehydrogenation (PMHS) reaction on the surface of the filter paper. A solution containing PMHS (10 wt $\%$ ) was added to a beaker containing $50 \mathrm{~g}$ of $\mathrm{n}$-hexane and Kastredt catalyst. Next, the filter paper was soaked in the modifier solution for approximately $2 \mathrm{~min}$, and then washed with $50 \mathrm{~mL}$ of $\mathrm{n}$-hexane three times to remove any solution ungrafted to the PMHS chain of the filter paper. The water contact angle was $131.8^{\circ}$. Existing methods usually dissolve hydrophobic modification reagents or modified nanomaterials in organic solvents, and then are sprayed on the surface of the material (Kim et al. 2013; Sheng et al. 2017; Guan et al. 2018). This type of method effectively achieves the hydrophobic modification of the surface of the paper, but there are still some problems. For example, using a large amount of organic solvents will cause damage to paper cultural relics as well as pollute the environment (Farré et al. 2010; Poggi et al. 2010; Amornkitbamrung et al. 2015; Balocco et al. 2016; Han et al. 2016). Therefore, it is of great importance to develop a simple, effective, and organic solvent-free method to prepare hydrophobic paper.

The gas-phase modification method has attracted widespread attention in the field of hydrophobic modification, due to its advantages of wide availability, convenient operation, and high yield (Aljumaily et al. 2018). Some hydrophobic modification methods using chemical vapor deposition deposit solid products on the surface of the substrate via gas phase or surface reactions, which increases the structure roughness of the surface. However, the post-processing of these methods still requires a great deal of organic solvents for hydrophobic modification (Rezaei et al. 2014; Zhang et al. 2018; Guo et al. 2019). The use of organic solvents will cause damage to the environment and experiment 
operators. As a result, the authors proposed a paper hydrophobic treatment method based on the chemical vapor deposition method without organic solvents. A small amount of hydrophobic modification reagent was formed into vapor in a confined space and then reacted with the active hydroxyl groups on the surface of paper, which quickly and simply underwent surface modification of the paper. The water contact angle reached $148^{\circ}$. Without other organic solvents, it effectively improves the protective performance of paper, and has good application prospects in the field of hydrophobic modification.

\section{EXPERIMENTAL}

\section{Equipment and Materials of Experiment}

(Heptadecafluoro-1,1,2,2-decyl)triethoxysilane (HFTTES) was purchased from Quzhou Dongming Chemical Co., Ltd. (Quzhou, China). Hexadecyltrimethoxysilane (HDTMS) and methyltrimethoxysilane (MTMS) were purchased from Shanghai Maclean Biochemical Technology Co., Ltd. (Shanghai, China). The red ink(G-5) was purchased from Chengguang Group Company (M \& G). The wood block was made of poplar. Whatman, grade 1 chromatography filter paper 3001-861 was purchased from Shanghai Bitai Biotechnology Co., Ltd. (Shanghai, China). The plasma treatment equipment was purchased from Shenzhen Dongxin Hi-Tech Automation Equipment Co., Ltd. (Shenzhen, China), which is a TS-PL02, a small plasma cleaning machine. Plasma is primarily composed of atoms, molecules, electrons, free radicals, and various active groups. During the plasma surface treatment process, when the plasma collides with the surface of the material, its energy will be transferred to the molecules and atoms on the surface of the material, thus generating a series of physical and chemical reactions to achieve the purpose of cleaning and modification. In this experiment, parameters were $300 \mathrm{~W}$ (power), parallel plate (reactor type), $-100 \mathrm{kPa}$ (pressure), $40 \mathrm{KHz} / 13.56 \mathrm{MHz}$ (supply frequency), air (plasma atmosphere).

There are two primary reactions that can occur between plasma and the surface of the material. One is a chemical reaction via free radicals, and the other is a physical reaction via plasma. The drying cabinet was purchased from Shanghai Kuntian Laboratory Instrument Co., Ltd. (Shanghai, China). The pressure-resistant reaction bottle, which had a volume of approximately $176.6 \mathrm{~cm}^{3}$, was purchased from Beijing Yixiubogu Biotechnology Co., Ltd. (Beijing, China) (as shown in ESI. S1).

\section{Preparation Method of the Hydrophobic Paper}

According to the experimental conditions, firstly, the filter paper was cut into a small $2 \mathrm{~cm} \times 10 \mathrm{~cm}$ piece, and then treated with plasma on both sides for $10 \mathrm{~min}$. Secondly, the filter paper was put in a pressure-resistant reaction flask and $10 \mu \mathrm{L}$ of HFTTES was dropped on the cap. Next, the paper was put into the drying cabinet at a temperature of 120 ${ }^{\circ} \mathrm{C}$ for $6 \mathrm{~h}$. Finally, the contact angle was measured between the surface of the sample and the water droplet using a $2 \mu \mathrm{L}$ droplet. Each sample was taken at five points to measure and calculate the average value (Yang and Deng 2008)

\section{Characterization of the Surface}

The surface morphology of the sample was observed via a Feina scanning electron microscope, model Phenom G6 pro, which was purchased from Reno Scientific Instruments Co., Ltd. (Shanghai, China). The contact angle between the sample and the 
water drop was measured with a SCI2000A contact angle measuring instrument, which was purchased from Beijing Global Hengda Technology Co., Ltd. (Beijing, China). The test drop was $2 \mu \mathrm{L}$ of pure water. Each sample was measured at 5 different positions, and the average value was taken.

\section{RESULTS AND DISCUSSION}

\section{The Principle of the Experiment}

The reaction process is shown in Fig. 1a. The hydrophobic modification reagent was dropped in a closed container with a piece of paper. The paper would be modified to form a hydrophobic surface under a certain reaction temperature and time. As a result, the writing did not get blurred after exposure to water. The experimental principle is shown in Fig. 1b. After plasma treatment, the hydroxyl group on the surface of the paper was activated (as shown in ESI S2). The hydroxyl group reacted with the ethoxy group to generate ethanol and volatilize. The hydrophobic $\mathrm{R}$ group and the silane chain were covalently grafted to inside the paper, so the paper was transformed from hydrophilic to hydrophobic.

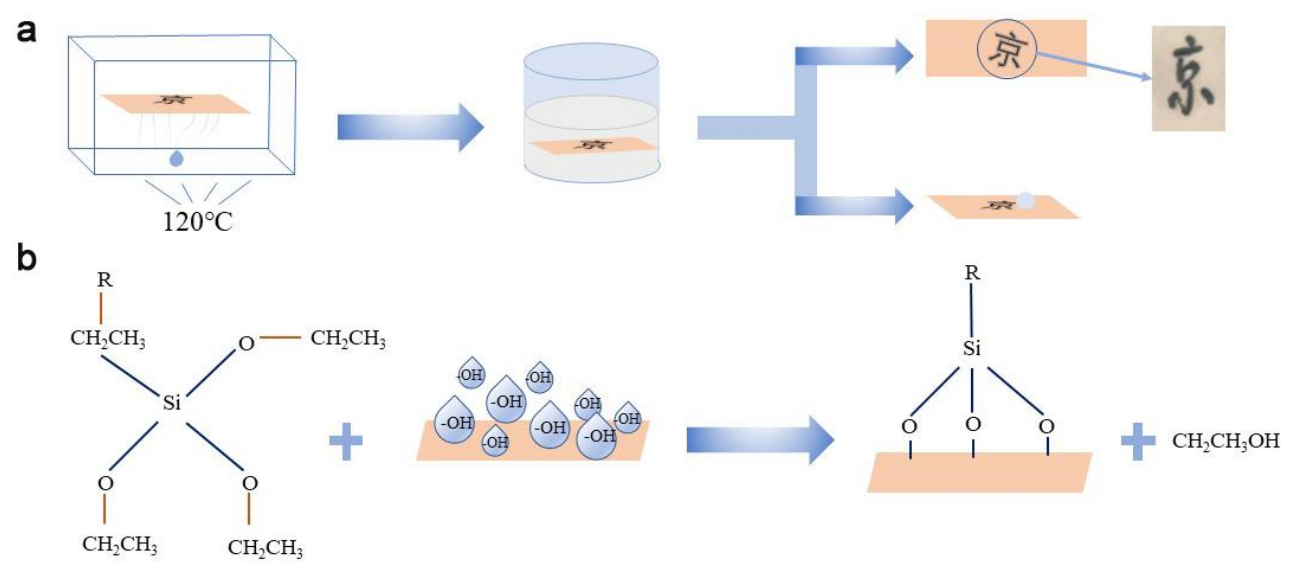

Fig. 1. The reaction principle diagram: (a) schematic diagram of the reaction process; and (b) schematic diagram of hydrophobic modification.

\section{The Optimization of the Experiment}

The effect of the reaction time on the hydrophobic property

This study explored the effect of the reaction time on the hydrophobic properties of the paper and conducted experimental optimization. The reaction time was set to $2 \mathrm{~h}, 4 \mathrm{~h}$, $6 \mathrm{~h}$, or $8 \mathrm{~h}$ during the preparation process. The relationship between the reaction time and the contact angle of the hydrophobic paper surface is shown in Fig. 2a. It can be seen from the Fig. 2 that the contact angle first increased and then decreased as the reaction time increased. When the reaction time reached $6 \mathrm{~h}$, the contact angle of the hydrophobic surface reached its maximum. Therefore, the optimal reaction time was $6 \mathrm{~h}$. As such, in the following experiments, the reaction time was $6 \mathrm{~h}$.

The effect of the plasma treatment time on the hydrophobic property

During the experiment process, the plasma treatment time was set to $1,3,6,10$, or 
12 min. The influence of the plasma treatment time on the contact angle is shown in Fig. $2 \mathrm{~b}$. The paper was treated with plasma to activate the hydroxyl groups on the surface of the paper, which can improve its hydrophilicity. As the plasma treatment time increased, the hydrophilic groups also gradually increased. However, this did not mean that the longer the plasma treatment time, the better the hydrophilicity. When the hydrophilic group was saturated, the hydrophilicity would no longer increase. If the treatment time continued to increase, the $\mathrm{C}-\mathrm{C}$ and $\mathrm{C}-\mathrm{H}$ bonds in the fiber molecules were broken, forming free radicals; then, free radicals react with each other to form crosslinks, which limited the penetration of water, thus decreasing the hydrophilicity (Deng et al. 2019). It could be seen in Fig. 2b that when the plasma treatment time was $10 \mathrm{~min}$, the hydrophilicity was the best and the activity of the paper surface was the highest as well. Then, the paper was modified with HFTTES ((heptadecafluoro-1,1,2,2-decyl)triethoxysilane). During this process, HFTTES was incorporated into the fiber structure to convert the hydrophilicity of the paper into hydrophobicity. Consequently, the optimal plasma treatment time was $10 \mathrm{~min}$.
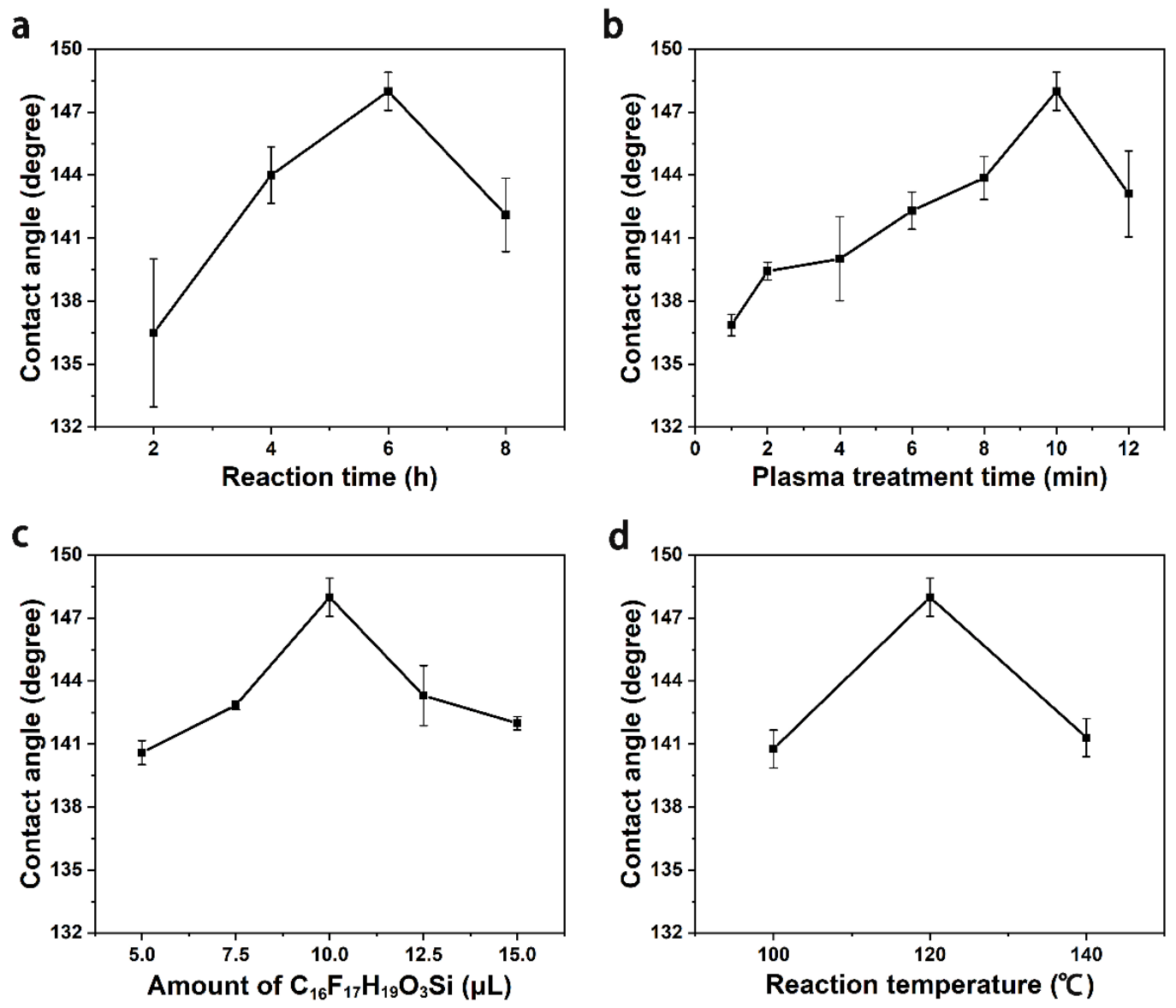

Fig. 2. The influence of the four variables on the contact angle of the hydrophobic paper surface in the experiment: (a) contact angles under different reaction time conditions; (b) contact angles under different plasma treatment time conditions; (c) contact angle with different amounts of (heptadecafluoro-1,1,2,2-decyl)triethoxysilane; and (d) contact angle under different reaction temperature conditions 


\section{The effect of the amount of (heptadecafluoro-1,1,2,2-decyl)triethoxysilane on the} hydrophobic property

In order to find the optimal amount of HFTTES, the amount of HFTTES in the process was $1,5,10,15$, or $20 \mu \mathrm{L}$. The relationship between the change in the amount of HFTTES and the contact angle of the hydrophobic paper is shown in Fig. 2c. This study utilized HFTTES to decrease the surface energy to construct a hydrophobic structure surface. After the hydrophobic groups were grafted onto the surface of the cellulose, the hydrophobicity of the paper was improved. It could be seen in Fig. 2c that the contact angle of the hydrophobic paper reached its maximum when the amount of HFTTES was $10 \mu \mathrm{L}$. When the amount of HFTTES was greater than $10 \mu \mathrm{L}$, the contact angle had little change from the contact angle at $10 \mu \mathrm{L}$. When the amount of HFTTES was 10,15 , and $20 \mu \mathrm{L}$, the contact angle was almost the same. It could be seen that the amount of HFTTES reached saturation at $10 \mu \mathrm{L}$. Therefore, the authors decided to adopt $10 \mu \mathrm{L}$ of HFTTES in the subsequent experiments. In addition, compared with other hydrophobic modification methods that used organic solvents, this method not only did not need to dissolve the hydrophobic modification solvent in an organic solvent, but also avoided the generation of harmful gases and waste, and as such was more environmentally friendly.

\section{The effect of the reaction temperature on the hydrophobicity}

The reaction temperature was set as a variable, and the other parameters were fixed values in order to study the effect of the reaction temperature on the hydrophobic properties of the paper. During the experiment process, the reaction temperature was set to 100,120 , or $140^{\circ} \mathrm{C}$. The relationship between the reaction temperature and the contact angle of the hydrophobic paper is shown in Fig. 2d. It can be seen in Fig. 2d that the contact angle reached its maximum when the temperature was $120{ }^{\circ} \mathrm{C}$. According to the measured contact angle value, it can be inferred that the most hydrophobic groups were grafted onto the paper at $120^{\circ} \mathrm{C}$.

It can be seen in Fig. 2 that when the plasma treatment time was $10 \mathrm{~min}$, the amount of HFTTES was $10 \mu \mathrm{L}$, the reaction time was $6 \mathrm{~h}$, and the reaction temperature was 120 ${ }^{\circ} \mathrm{C}$, which were the optimal preparation conditions, the contact angle reached its maximum value. As such, the authors designed 3 sets of parallel experiments (Fig. S1) in order to prove that this was not an accidental phenomenon. After taking the average value, the contact angle was $148^{\circ}$.

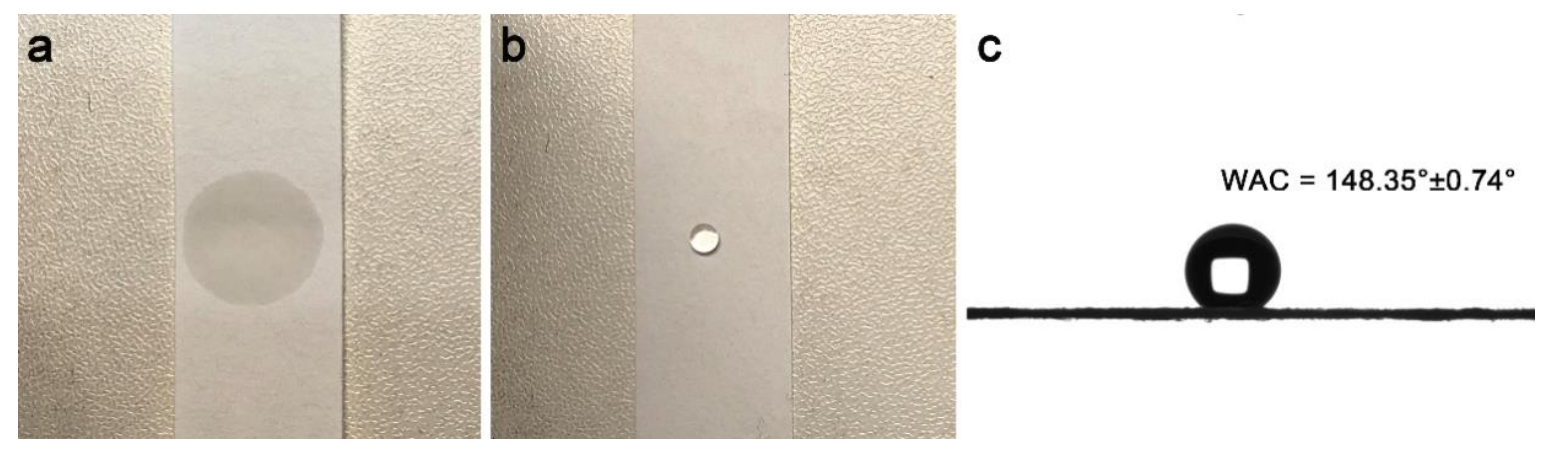

Fig. 3. Photos of the water droplets on the surface of filter paper: (a) before modification; (b) after modification; and (c) measurement of the hydrophobic paper contact angle under optimal preparation conditions 
Figure $3 \mathrm{a}$ shows a photo of the water droplets on the unmodified filter paper. Obviously, the water droplets were quickly absorbed by the filter paper. The paper after the hydrophobic treatment clearly had hydrophobic properties. The contact angle with water reached approximately $148^{\circ}$ (Fig. 3b and 3c), which showed the paper changed from hydrophilic to hydrophobic. As shown in Fig. 4, which compared the fiber structure before and after the paper modification, it was found that there was no distinct change, which showed the fiber structure inside the paper was not damaged after paper hydrophobic modification via this method. Supposing that this method was applied to the protection of paper cultural relics, the paper cultural relics could be given waterproof properties to deal with the damage caused by changes in environmental humidity.

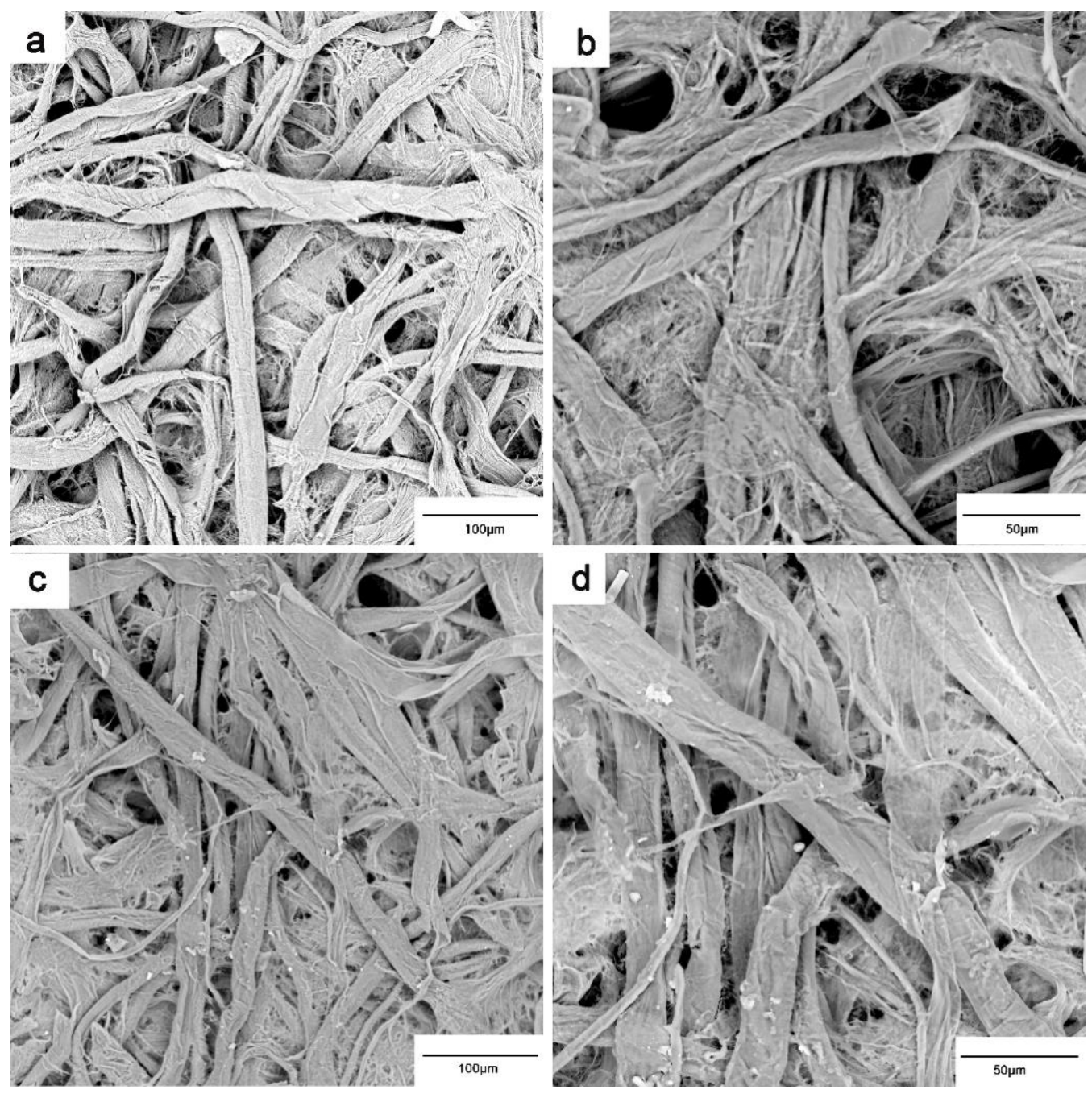

Fig. 4. SEM images before and after filter paper modification: (a) 600x magnification without modification; (b) 1200x magnification without modification; (c) 600x magnification after modification; and (d) 1200x magnification after modification

\section{Analysis of the Easy Cleaning Performance}

Red ink (Fig. 5) and oil (Fig. S3) were dripped on the hydrophobic paper, and then wiped with a paper towel for test the easy cleaning performance of the paper. It was found that no obvious stains remained on the surface, which showed that the paper had good hydrophobic and oleophobic properties and had a certain degree of easy cleaning. 

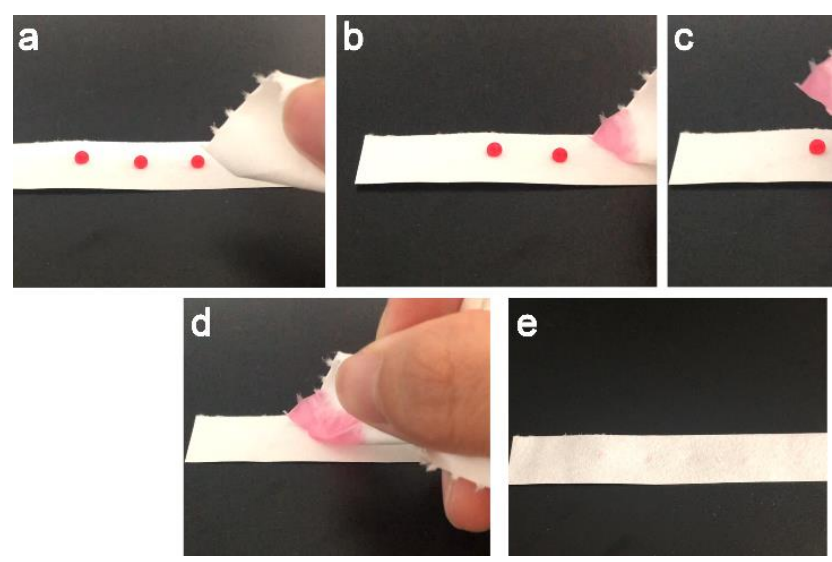

Fig. 5. Easy cleanability test of the hydrophobic filter paper

\section{Application of this Method}

The authors experimented by writing in pen on the paper in order to verify the practicability of this method. As commonly known, when paper with pen writing is exposed to water or other liquids, the writing will become blurred and illegible. As shown in Fig. 6, the filter paper with pen writing without the hydrophobic treatment was soaked in water and taken out after 2 min. Afterwards, the writing was washed away by the water and became blurred (Fig. 6b). The same was done for the treated hydrophobically paper. As shown in Fig. 6d, the writing was still clearly identifiable. The authors also carried out the same experimental treatment (Fig. S1) on A4 paper, which had the same experimental effect as filter paper.
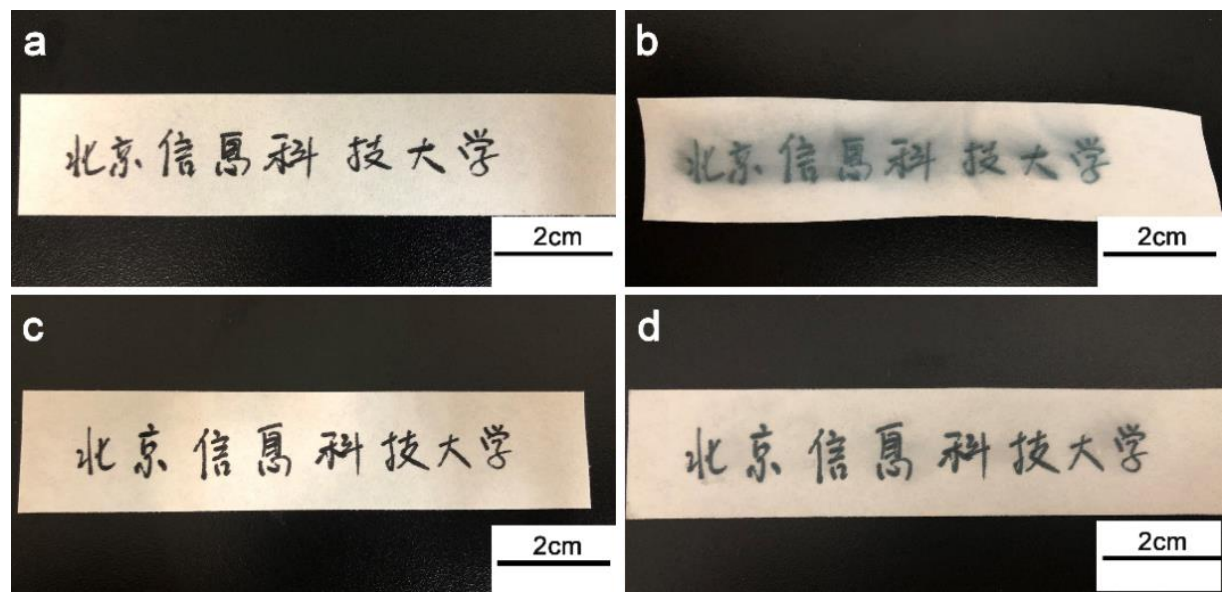

Fig. 6. Test of the filter paper with pen writing: (a) filter paper strips without the hydrophobic treatment; (b) filter paper strips without the hydrophobic treatment soaked in water for 2 min and then taken out; (c) filter paper strips with the hydrophobic treatment; and (d) the hydrophobically treated filter paper soaked in water for 2 min and then taken out

A digital microscope (SK2300VGA, Shenzhen Saike Digital Technology Development Co. Ltd., Shenzehn, China) was also used to characterize the filter paper. Taking the word “京” as an example, A4 paper that had not been soaked in water (marked as No. 1), A4 paper that had not been soaked in water without the hydrophobic treatment (marked as No. 2), and A4 paper (marked as No. 3) soaked in water with the hydrophobic 
treatment were compared (Fig. 7). Under the digital microscope, it can be clearly seen that the handwriting on the No. 2 paper was severely washed away by the water, and the handwriting was blurred. After the No. 3 paper (Fig. 7f) was soaked, the handwriting was still clearly visible. It can be seen that the writing would not become blurred even if the paper with hydrophobic treatment was exposed to water.

The above experiments showed that the paper treated by this method had a certain degree of hydrophobicity as well as easy cleaning properties. Most important of all, this treatment did not cause damage to the fiber structure inside the paper. As such, the authors hope that this method can be applied to the hydrophobic protection of paper cultural relics, so that the paper cultural relics have watertightness and moisture resistance properties to delay the aging of paper cultural relics to a certain extent.
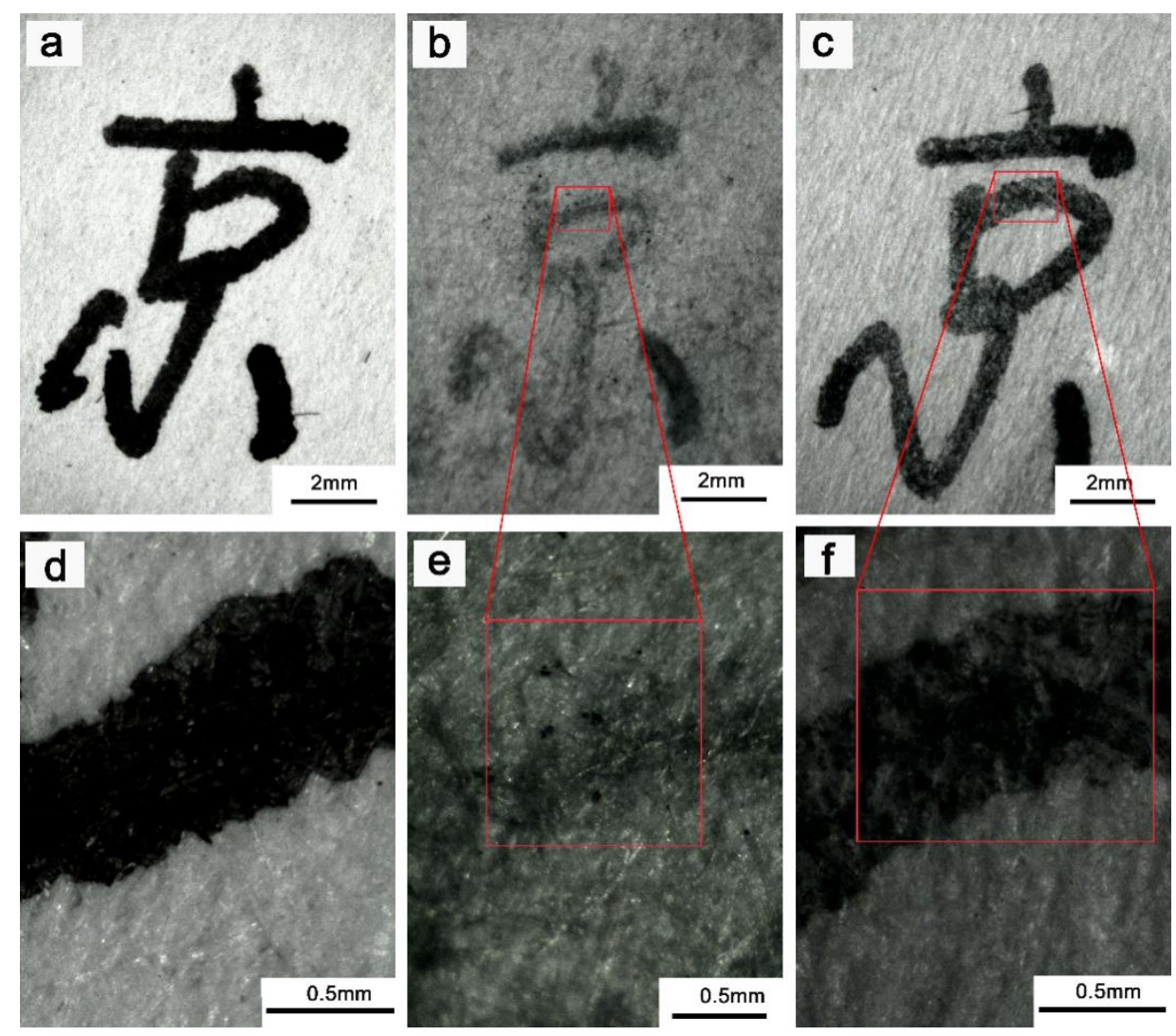

Fig. 7. The A4 paper with pen writing under a digital microscope: (a) the unsoaked paper; (b) the soaked paper without the hydrophobic treatment; (c) the hydrophobically treated paper after soaking; (d) an enlarged version of (a); (e) an enlarged version of (b); and (f) an enlarged version of (c)

\section{Versatility of this Method}

Since wooden cultural relics also need protection, this method was applied to other substrates, i.e., wood blocks. After the hydrophobization treatment, the hydrophobic property of the wood block was tested by measuring the contact angle with droplets. First, the wood blocks were placed on the contact angle measuring instrument. Next, the water contact angles of 5 different positions were measured, with a water droplet size of approximately $2 \mu \mathrm{L}$. Then, the average value was calculated. The measurement results are shown in Fig. S1. It can be clearly seen in Fig. S1 that the performance of the wood block 
transformed from hydrophilic to hydrophobic after the hydrophobization treatment. The contact angle was approximately $141^{\circ}$. It was found that the hydrophobic performance of the wood block was effectively improved after the treatment via this method, which demonstrated that this method had a certain versatility for different substrates. The red ink droplets were dropped on the hydrophobic small wooden block, and after wiping with a paper towel, the ink stain did not remain on the small wooden block. This indicated that the small wooden block was easy to clean (Fig. S4).

Different paper materials have different requirements for hydrophobic groups. Therefore, this study tried other hydrophobically modified silane reagents, i.e., hexadecyltrimethoxysilane and methyltrimethoxysilane. The paper was processed in the same way as above for these reagents. Next, the experimental effect was observed by measuring the contact angle at five different positions and averaged. The contact angles were $140.78^{\circ}$ and $142.71^{\circ}$, respectively (Fig. S1), which indicated that this method was also applicable to other hydrophobic modifications reagents.

Compared with some complex methods reported in the past, this method improves the operability of the experiment while avoiding the need for organic solvents, thereby minimizing costs, and reducing safety and environmental issues. The preparation methods shown in Table 1 all used organic solvents, while the method proposed by this study only used a small amount of organic reagent $(10 \mu \mathrm{L})$, without organic solvents. The use of organic solvents will cause certain harm to the environment and laboratory personnel.

Table 1. Comparison of Several Hydrophobic Preparation Methods

\begin{tabular}{|c|c|c|c|c|c|}
\hline $\begin{array}{l}\text { Hydrophobic } \\
\text { Treatment } \\
\text { Method }\end{array}$ & $\begin{array}{l}\text { Organic } \\
\text { Solvents }\end{array}$ & $\begin{array}{l}\text { Modification } \\
\text { Reagents }\end{array}$ & $\begin{array}{c}\text { Amount of } \\
\text { Hydrophobic } \\
\text { Reagent }\end{array}$ & $\begin{array}{c}\text { Amount of } \\
\text { Hydrophobic } \\
\text { Reagent } \\
\left(\mathrm{mL} / \mathrm{cm}^{2}\right)\end{array}$ & $\begin{array}{l}\text { Contact } \\
\text { Angle }\left(^{\circ}\right)\end{array}$ \\
\hline $\begin{array}{c}\text { Coating } \\
\text { method } \\
\text { (Zhang et al. } \\
\text { 2012) } \\
\end{array}$ & $\begin{array}{l}\text { Tetrahydrofuran; } \\
\text { acetone }\end{array}$ & Octadecylamine & $15 \mathrm{~mL}$ & 1.67 & 156 \\
\hline $\begin{array}{c}\text { Spray } \\
\text { method } \\
\text { (Li et al. } \\
2012)\end{array}$ & $\begin{array}{l}\text { Dehydrated } \\
\text { toluene }\end{array}$ & $\begin{array}{l}\text { Octadecyl } \\
\text { trichlorosilane }\end{array}$ & $1 \mathrm{~mL}$ & 0.035 & 163 \\
\hline $\begin{array}{l}\text { Impregnation } \\
\text { method } \\
\text { (Medina- } \\
\text { Sandoval et } \\
\text { al. 2018) }\end{array}$ & $\begin{array}{c}\text { Dichloromethane; } \\
\text { N-heptane }\end{array}$ & $\begin{array}{c}\text { Hexadecyl- } \\
\text { trimethoxysilane }\end{array}$ & $>3.5 \mathrm{~mL}$ & 0.067 & 120 \\
\hline $\begin{array}{l}\text { Impregnation } \\
\text { method } \\
\text { (Liu et al. } \\
\text { 2018) }\end{array}$ & N-hexane & $\begin{array}{c}\text { Polymethyl- } \\
\text { hydrogensiloxane }\end{array}$ & $6.25 \mathrm{~mL}$ & 0.22 & 131.8 \\
\hline This study & None & $\begin{array}{c}\text { (Heptadecafluoro- } \\
\text { 1,1,2,2-decyl) } \\
\text { triethoxysilane }\end{array}$ & $10 \mu \mathrm{L}$ & 0.001 & 148.35 \\
\hline
\end{tabular}




\section{CONCLUSIONS}

1. In order to realize the hydrophobic modification of paper, this work proposed an experimental method which did not rely on organic solvents. Under optimal experimental conditions, the contact angle of the hydrophobic paper was $148^{\circ}$.

2. The soaking experiments, i.e., paper with pen writing being dipped in water, showed that the writing on the paper treated by this method was still clear and recognizable after it was exposed to water.

3. The antifouling experiments showed that there was no obvious remainder on the paper after wiping away the red ink and oil, which verified that the hydrophobic paper had easy cleaning performance.

4. This study also conducted experiments using two other hydrophobic reagents and other substrates to show that this method had good versatility.

5. The process of this method is simple, is friendly to environment, and only uses a small amount of hydrophobic modification reagent $(10 \mu \mathrm{L})$, as well as no organic solvents. This technical idea was proposed for the hydrophobic and antifouling protection of paper cultural relics.

\section{ACKNOWLEDGMENTS}

The authors wish to express their appreciation to the Scientific Research Level Enhancement Special Project (Key Research and Cultivation Project No. 2021YJPY239) and the Beijing Key Laboratory for Sensors of the Beijing Information Science and Technology University. This work was supported by the National Natural Science Foundation of China (Grant No. 61601037 and Grant No. 61901042), the National Key R\&D Program of China (Grant No. 2018YFA0106900), the Research Foundation for Youth Scholars of Beijing Technology and Business University (Grant No. QNJJ2020-04), and the Open Research Fund Program of the Institute of Regulatory Science (Beijing Technology and Business University).

\section{REFERENCES CITED}

Aljumaily, M. M., Alsaadi, M. A., Das, R., Hamid, S. B. A., Hashim, N. A., AlOmar, M. K., Alayan, H. M., Novikov, M., Alsalhy, Q. F., and Hashim, M. A. (2018). "Optimization of the synthesis of superhydrophobic carbon nanomaterials by chemical vapor deposition," Scientific Reports 8(1), 1-12. DOI: 10.1038/s41598-01821051-3

Amornkitbamrung, L., Mohan, T., Hribernik, S., Reichel, V., Faivre, D., Gregorova, A., Engel, P., Kargl, R., and Ribitsch, V. (2015). "Polysaccharide stabilized nanoparticles for deacidification and strengthening of paper," RSC Advances 5(42), 32950-32961. DOI: $10.1039 / C 4 R A 15153 D$

Balocco, C., Petrone, G., Maggi, O., Pasquariello, G., Albertini R., and Pasquarella C. (2016). "Indoor microclimatic study for cultural heritage protection and preventive conservation in the Palatina Library," Journal of Cultural Heritage 22, 956-967. DOI: 
10.1016/j.culher.2016.05.009

Chen, Q., Wen, W.-Y., Qiu, F.-X., Xu, J.-C., Yu, H.-Q., Chen, M.-L., and Yang, D.-Y. (2016). "Preparation and application of modified carboxymethyl cellulose Si/polyacrylate protective coating material for paper relics," Chemical Papers 70(7), 946-959. DOI: 10.1515/chempap-2016-0029

Deng, J. W., Guo, Y., Xu, L. Y., Zhang, R. Y., and Yu, J. Y. (2019). "Effects of lowpressure plasma process parameters on the preparation of superhydrophobic polyester fabric," Shanghai Textile Science \& Technology 47(436), 56-61.

Farré, M., Pérez, S., Gonçalves, C., Alpendurada, M. F., and Barceló, D. (2010). “Green analytical chemistry in the determination of organic pollutants in the aquatic environment," TrAC Trends in Analytical Chemistry 29(11), 1347-1362. DOI: 10.1016/j.trac.2010.07.016

Guan, Y., Yu, C., Zhu, J., Yang, R., Li, X., Wei, D., and Xu, X. (2018). “Design and fabrication of vapor-induced superhydrophobic surfaces obtained from polyethylene wax and silica nanoparticles in hierarchical structures," RSC Advances 8(44), 2515025158. DOI: $10.1039 / \mathrm{C} 8 \mathrm{RA01666F}$

Guo, X.-J., Xue, C.-H., Sathasivam, S., Page, K., He, G., Guo, J., Promdet, P., Heale, F. L., Carmalt, C. J., and Parkin, I. P. (2019). "Fabrication of robust superhydrophobic surfaces via aerosol-assisted CVD and thermo-triggered healing of superhydrophobicity by recovery of roughness structures," Journal of Materials Chemistry A 7(29), 17604-17612. DOI: 10.1039/C9TA03264A

Han, S., Sun, Q., Zheng, H., Li, J. and Jin, C. (2016). "Green and facile fabrication of carbon aerogels from cellulose-based waste newspaper for solving organic pollution," Carbohydrate Polymers 136, 95-100. DOI: 10.1016/j.carbpol.2015.09.024

Kim, H.-M., Sohn, S., and Ahn, J. S. (2013). "Transparent and super-hydrophobic properties of PTFE films coated on glass substrate using RF-magnetron sputtering and Cat-CVD methods," Surface and Coatings Technology 228(S1), S389-S392. DOI: 10.1016/j.surfcoat.2012.05.085

Lazaridis, M., Katsivela, E., Kopanakis, I., Raisi, L., Mihalopoulos, N., and Panagiaris G. (2018). "Characterization of airborne particulate matter and microbes inside cultural heritage collections," Journal of Cultural Heritage 30, 136-146. DOI: 10.1016/j.culher.2017.09.018

Li, J., Wan, H., Ye, Y., Zhou, H., and Chen, J. (2012). "One-step process to fabrication of transparent superhydrophobic $\mathrm{SiO}_{2}$ paper," Applied Surface Science 261, 470-472. DOI: 10.1016/j.apsusc.2012.08.034

Li, Q., Yan, Y., Yu, M., Song, B., Shi, S., and Gong, Y. (2016). "Synthesis of polymeric fluorinated sol-gel precursor for fabrication of superhydrophobic coating," Applied Surface Science 367, 101-108. DOI: 10.1016/j.apsusc.2016.01.155

Li, X., Chen, G., Ma, Y., Feng, L., Zhao, H., Jiang, L., and Wang, F. (2006). "Preparation of a super-hydrophobic poly (vinyl chloride) surface via solvent-nonsolvent coating," Polymer 47(2), 506-509. DOI: 10.1016/j.polymer.2005.08.097

Li, X., Yang, J., Lv, K., Papadopoulos, P., Sun, J., Wang, D., Zhao, Y., Chen, L., Wang, D., Wang, Z., et al. (2021). "Salvinia-like slippery surface with stable and mobile water/air contact line," National Science Reviews 8(5), 153-185. DOI: $10.1093 / \mathrm{nsr} / \mathrm{nwaa} 153$

Li, X., Yu, X., Cheng, C., Deng, L., Wang, M., and Wang, X. (2015). “Electrospun superhydrophobic organic/inorganic composite nanofibrous membranes for membrane distillation," ACS Applied Materials \& Interfaces 7(39), 21919-21930. 
DOI: 10.1021/acsami.5b06509

Li, X.-M., Reinhoudt, D., and Crego-Calama, M. (2007). "What do we need for a superhydrophobic surface? A review on the recent progress in the preparation of superhydrophobic surfaces," Chemical Society Reviews 36(8), 1350-1368. DOI: 10.1039/B602486F

Liu, L., Zhang, L., Zhang, B., and Hu, Y. (2019). "A comparative study of reinforcement materials for waterlogged wood relics in laboratory," Journal of Cultural Heritage 36, 94-102. DOI: 10.1016/j.culher.2018.08.002

Liu, Z., Yu, J., Lin, W., Yang, W., Li, R., Chen, H., and Zhang, X. (2018). "Facile method for the hydrophobic modification of filter paper for applications in water-oil separation," Surface and Coatings Technology 352, 313-319. DOI: 10.1016/j.surfcoat.2018.08.026

Medina-Sandoval, C. F., Valencia-Dávila, J. A., Combariza, M. Y., and Blanco-Tirado, C. (2018). "Separation of asphaltene-stabilized water in oil emulsions and immiscible oil/water mixtures using a hydrophobic cellulosic membrane," Fuel 231, 297-306. DOI: 10.1016/j.fuel.2018.05.066

Musikavanhu, B., Hu, Z., Dzapata, R. L., Xu, Y., Christie, P., Guo, D., and Li J. (2019). "Facile method for the preparation of superhydrophobic cellulosic paper," Applied Surface Science 496, 1-9. DOI: 10.1016/j.apsusc.2019.143648

Poggi, G., Giorgi, R., Toccafondi, N., Katzur, V., and Baglioni, P. (2010). "Hydroxide nanoparticles for deacidification and concomitant inhibition of iron-gall ink corrosion of paper," Langmuir 26(24), 19084-19090. DOI: 10.1021/la1030944

Reyden, D. V. D. (1992). "Recent scientific research in paper conservation," Journal of the American Institute for Conservation 31(1), 117-138. DOI: 10.1179/019713692806156394

Rezaei, S., Manoucheri, I., Moradian, R., and Pourabbas, B. (2014). "One-step chemical vapor deposition and modification of silica nanoparticles at the lowest possible temperature and superhydrophobic surface fabrication," Chemical Engineering Journal 252, 11-16. DOI: 10.1016/j.cej.2014.04.100

Sheng, J., Xu, Y., Yu, J., and Ding, B. (2017). "Robust fluorine-free superhydrophobic amino-silicone oil/ $/ \mathrm{SiO}_{2}$ modification of electrospun polyacrylonitrile membranes for waterproof-breathable application," ACS Applied Materials \& Interfaces 9(17), 15139-15147. DOI: 10.1021/acsami.7b02594

Wang, N., Xiong, D., Pan, S., Deng, Y., Shi, Y., and Wang, K. (2016).

"Superhydrophobic paper with superior stability against deformations and humidity," Applied Surface Science 389, 354-360. DOI: 10.1016/j.apsusc.2016.07.110

Wen, Q. Y. (2020). "Research on the application of preventive protection of paper cultural relics in the collection - taking Wenzhou Museum as an example," Wenzhou Cultural Relics 01, 79-90.

$\mathrm{Xu}, \mathrm{D}$. (2018). "Temperature and humidity control in the aging process of paper cultural relics," Cultural Heritage Identification and Appreciation 07, 124-125.

Xu, J., Zhang, T., Zhang, X., Jiang, Y., Yang, D., Qiu, F., and Yu, Z. (2020). "Preparation of polymeric material containing UV absorber for application in paperbased relics protection," Polymer-Plastics Technology and Materials 59(5), 536-545. DOI: $10.1080 / 25740881.2019 .1669651$

Xue, C.-H., Li, Y.-R., Zhang, P., Ma, J.-Z., and Jia, S.-T. (2014). "Washable and wearresistant superhydrophobic surfaces with self-cleaning property by chemical etching of fibers and hydrophobization," ACS Applied Materials \& Interfaces 6(13), 10153- 
10161. DOI: 10.1021/am501371b

Yang, H., and Deng, Y. (2008). "Preparation and physical properties of superhydrophobic papers," J. Colloid Interf. Sci. 325(2), 588-593.

Zervos, S., and Alexopoulou, I. (2015). "Paper conservation methods: A literature review," Cellulose 22, 2859-2897. DOI: 10.1007/s10570-015-0699-7

Zhang, C., Huang, Y., Zhang, H., Ye, Z., Liu, P., Wang, S., Zhang, Y., and Tang, Y. (2020). "Selectively functionalized zeolite NaY composite materials for highefficiency multiple protection of paper relics," Industrial \& Engineering Chemistry Research 59(24), 11196-11205. DOI: 10.1021/acs.iecr.0c00635

Zhang, F., Shi, Z., Xu, C., Huo, D., Zhang, W., and Peng, C. (2018). "Self-fibering growth in the soot-templated CVD coating of silica on mesh for efficient oil/water separation," Materials \& Design 154, 370-377. DOI: 10.1016/j.matdes.2018.05.038

Zhang, M., Wang, C., Wang, S., Shi, Y., and Li, J. (2012). "Fabrication of coral-like superhydrophobic coating on filter paper for water-oil separation," Applied Surface Science 261, 764-769. DOI: 10.1016/j.apsusc.2012.08.097

Article submitted: September 29, 2021; Peer review completed: November 6, 2021; Revised version received: November 17, 2021; Accepted: November 18, 2021;

Published: November 22, 2021.

DOI: 10.15376/biores.17.1.384-399 


\section{APPENDIX}

\section{Supplemental Material}

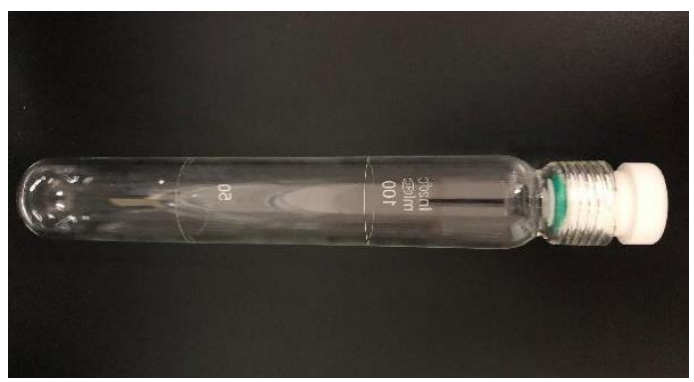

Fig. S1. The physical drawing of pressure-resistant reaction bottle

(a)

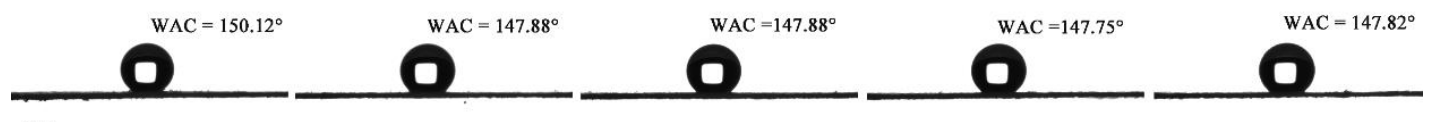

(b)

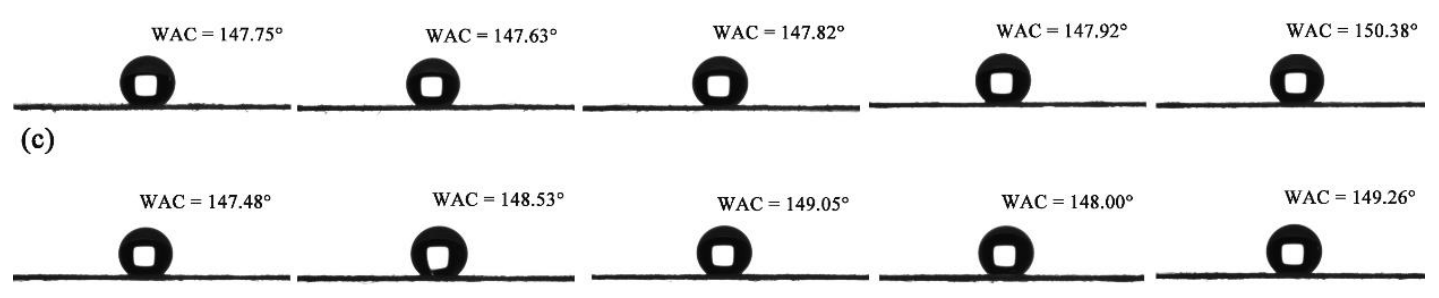

Fig. S2. The three groups are parallel experiments (a) Take the contact angle measured at 5 positions for the No. 1 sample of the parallel experiment (b) Take the contact angle measured at 5 positions for the No. 2 sample of the parallel experiment (c) Take the contact angle measured at 5 positions for the No. 3 sample of the parallel experiment.
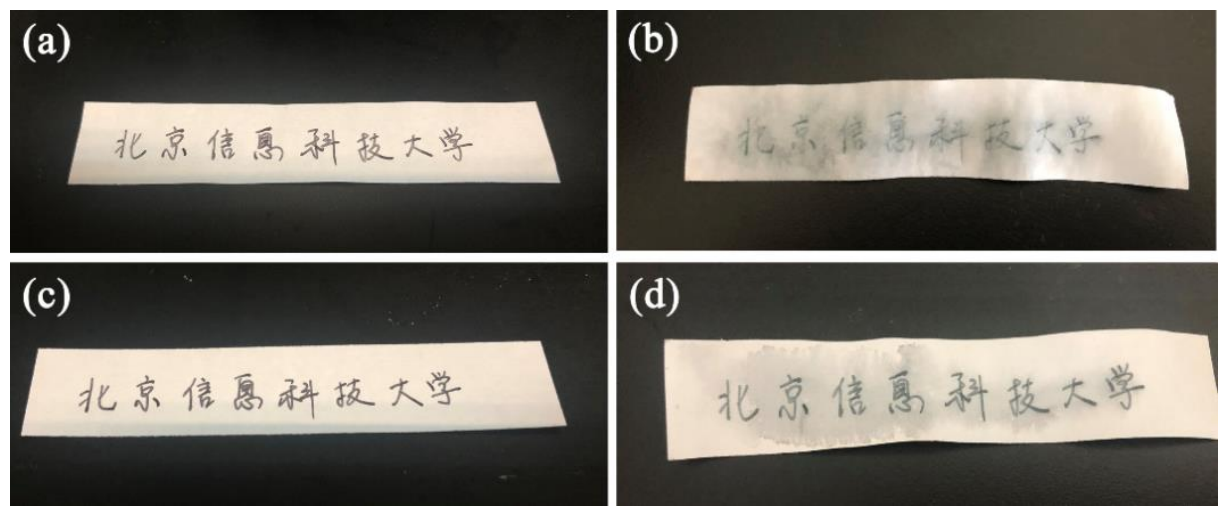

Fig. S3. Water immersion test on A4 paper with pen writing (a) A4 paper strip without hydrophobic treatment (c) the A4 paper strip was soaked without hydrophobic treatment in water for 2 minutes and took out (b) A4 paper strip with hydrophobic treatment (d) The hydrophobically treated A4 paper strip was soaked in water for 2 minutes and took out. 

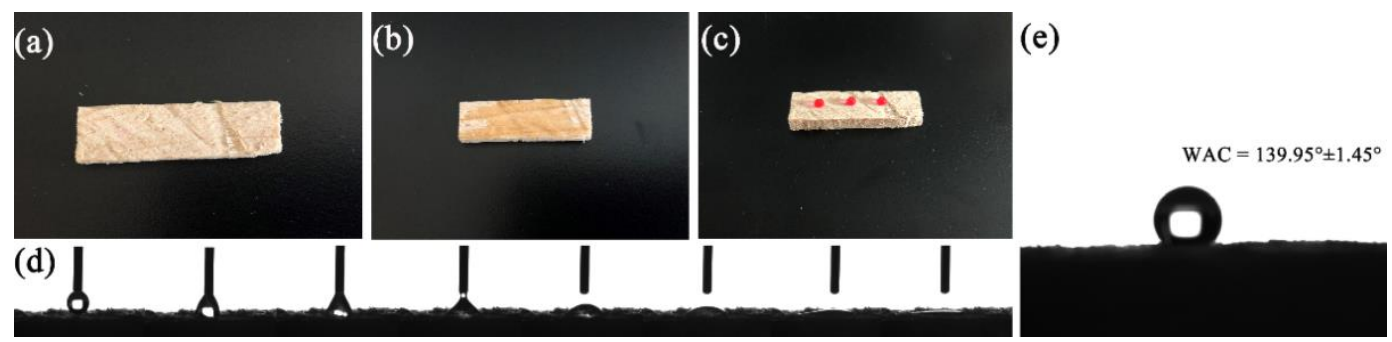

Fig. S4. The physical image of the wood block before and after modification and the measurement of the contact angle after modification (a) Wood block before modification (b) Hydrophilicity of wood block before modification (c) Modified wood block (d) Test of hydrophilicity of wooden blocks before modification (e) Measurement of contact angle between wood block and water after modification.

(a)

(b)

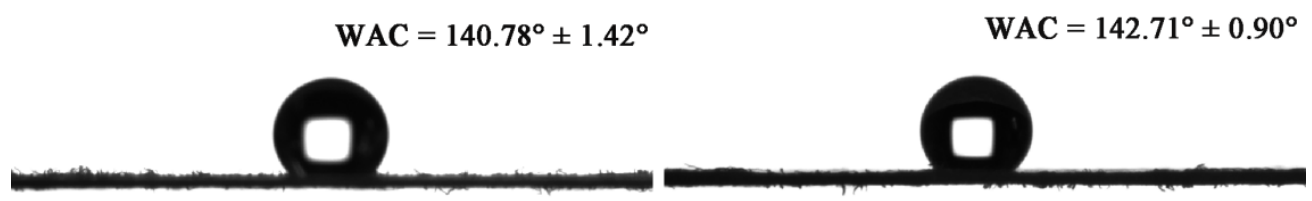

Fig. S5. After treatment with hexadecyltrimethoxysilane and methyltrimethoxysilane, the measurement of the contact angle between paper and water droplets (a) Contact angle measurement after treatment with hexadecyltrimethoxysilane (b) Contact angle measurement after treatment with methyltrimethoxysilane. 